\title{
SUBTIPOS MOLECULARES DE PML/RAR $\alpha$ EN PACIENTES CON LEUCEMIA PROMIELOCÍTICA AGUDA
}

\author{
María del Carmen Castro-Mujica ${ }^{1,2, a}$, Yasser Sullcahuamán-Allende ${ }^{1, b}$.
}

\author{
RESUMEN
}

El objetivo fue describir la frecuencia de los subtipos moleculares de PML/RARa en pacientes con leucemia promielocítica aguda (LPA) y su distribución según grupo de riesgo de recaída y citomorfología. Se realizó una serie de casos que incluyó a cincuenta pacientes registrados en el Instituto Nacional de Enfermedades Neoplásicas (INEN), durante el periodo 2010-2012, con diagnóstico molecular de LPA PML/RARa y subtipos bcr1, bcr2 y bcr3 por reacción en cadena de la polimerasa con transcriptasa reversa (RT-PCR). El subtipo bcr1 fue el más frecuente (62\%). Los pacientes con riesgo de recaída intermedio y morfología hipergranular fueron, en su mayoría, bcr1 (70\%) y todos los que poseían riesgo de recaída alto y morfología hipogranular fueron bcr3. Se concluye que en la población estudiada hay un predomino del subtipo bcr1 y que existen diferencias en la distribución de los subtipos bcr1 y bcr3 según el grupo de riesgo de recaída y citomorfología.

Palabras clave: Leucemia promielocítica aguda; Fusión génica; Reacción en Cadena de la polimerasa de transcriptasa inversa (fuente: DeCS BIREME).

\section{MOLECULAR SUBTYPES OF PML/RAR $\alpha$ IN PATIENTS WITH ACUTE PROMYELOCYTIC LEUKEMIA}

\section{ABSTRACT}

The objective was to describe the frequency of molecular subtypes of PML/RARa in patients with acute promyelocytic leukemia (APL) and their distribution according to risk of recurrence and cytomorphology. A case series was carried out, including fifty patients registered at the National Institute of Neoplastic Diseases (INEN) during 2010-2012, with molecular diagnosis of APL PML/RAR a and bcr1, bcr2 and bcr3 subtypes by reverse-transcription polymerase chain reaction (RT-PCR). Bcr1 subtype was the most frequent (62\%). Most patients with an intermediate risk of recurrence and hypergranular morphology were bcr1 (70\%), while all patients with high risk of recurrence and hypogranular morphology were bcr3. A predominance of bcr1 subtype among the population studied can therefore be concluded, as well as the fact that there are differences in the distribution of bcr1 and bcr3 subtypes according to recurrence risk group and cytomorphology.

Key words: Leukemia, promyelocytic, acute; Gene fusion; Reverse transcriptase polymerase chain reaction (source: MeSH NLM).

\section{INTRODUCCIÓN}

La leucemia promielocítica aguda (LPA), representa el $5-8 \%$ de las leucemias mieloides agudas (LMA) a nivel mundial, y el $20-28 \%$ en países latinoamericanos, predominando en adultos jóvenes (1-4). Anualmente la LPA representa el $22 \%$ del total de casos de LMA registrados en el Instituto Nacional de Enfermedades Neoplásicas (INEN) del Perú ${ }^{(5)}$. La LPA se clasifica citomorfológicamente según las características de los promielocitos leucémicos (hipergranular o típica (M3) e hipogranular o variante (M3V)) ${ }^{(6)}$, y por su riesgo de recaída (alto, intermedio o bajo) de acuerdo con los valores de leucocitos y plaquetas al diagnóstico (7).

El 95\% de las LPA se deben a la traslocación $\mathrm{t}(15 ; 17)$ (q24.1;q21.2) resultando la fusión génica PML/RARa que codifica una proteína híbrida que bloquea la diferenciación de los promielocitos (8). Esta fusión presenta tres subtipos moleculares según el punto de corte en el gen PML en el cromosoma $15^{(9)}$ (Figura 1), si sucede en el intrón 6 será isoforma L (Long) o bcr1, en

\footnotetext{
Unidad de Genética y Biología Molecular, Instituto Nacional de Enfermedades Neoplásicas. Lima, Perú.

Universidad Peruana Cayetano Heredia. Lima, Perú.

Médico cirujano, ${ }^{\mathrm{b}}$ médico genetista

Recibido: 06-01-13 Aprobado: 06-03-13
}

Citar como: Castro-Mujica MC, Sullcahuamán-Allende Y. Subtipos moleculares de PML/RARa en pacientes con leucemia promielocítica aguda. Rev Peru Med Exp Salud Publica. 2013;30(1):37-40. 

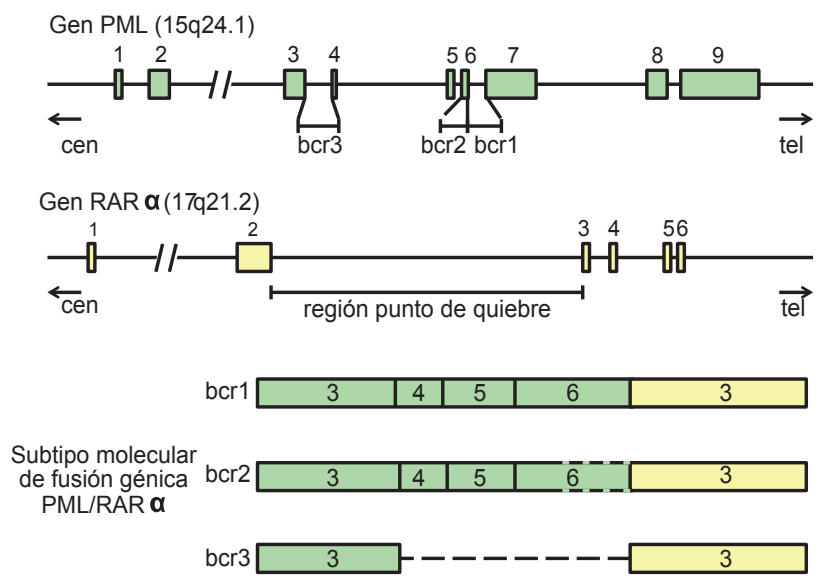

Figura 1. Representación de subtipos moleculares bcr1, bcr2 y bcr3 según punto de corte en el gen PML.

Modificado de "Van Dongen JJM et al. Standardized RT-PCR analysis of fusion gene transcripts from chromosome aberrations in acute leukemia for detection of minimal residual disease. Leukemia. 1999" ${ }^{(18)}$ con permiso del autor.

el exón 6 será isoforma $V$ (Variable) o bcr2 y en el intrón 3 será isoforma $\mathrm{S}$ (Short) o bcr3. La isoforma bcr1 es la más frecuente en la población latina ${ }^{(4,10-12)}$ y se asocia a la morfología hipergranular de los promielocitos. La isoforma bcr3 es la más frecuente en niños ${ }^{(13)}$, en el tipo hipogranular (14) y se asocia a hiperleucocitosis, coagulopatía hemorrágica, síndrome ATRA y una mayor prevalencia de mutaciones FLT3-ITD (FMSlike tyrosine kinase-3 in tándem duplication) ${ }^{(15)}$ que le confiere mal pronóstico a la enfermedad. El 5\% restante de casos de LPA corresponde a variantes debido a la fusión del gen RAR $\alpha$ con otros genes distintos a PML.

La reacción en cadena de la polimerasa por transcriptasa reversa (RT-PCR) realiza el diagnóstico definitivo de LPA tras detectar la fusión PML/RARa, siendo la prueba de oro para determinar el subtipo molecular e inferir el pronóstico de la enfermedad al momento del diagnóstico (16). Actualmente, en la supervisión de la enfermedad mínima residual se utiliza el PCR en tiempo real (RQ-PCR) cuya principal ventaja frente al RT-PCR es la cuantificación de transcriptos obtenidos de la fusión génica PML/RARa, útil en el seguimiento de la LPA ${ }^{(17)}$.

El objetivo del presente trabajo es describir el perfil molecular de la LPA PML/RARa en los pacientes que han sido registrados por primera vez en la Unidad de Genética y Biología Molecular (UGBM) del INEN desde el 2010 al 2012. No hemos encontrado estudios genéticos sobre LPA en el Perú, por lo que permitiremos ampliar la descripción de la frecuencia de los subtipos moleculares bcr1, bcr2 y bcr3 de la fusión génica PML/RARa y su distribución de acuerdo al sexo, edad, características citomorfológicas, y grupos de riesgo de recaída en nuestra población.

\section{EL ESTUDIO}

Se realizó un estudio observacional descriptivo tipo serie de casos a partir del registro de estudios moleculares de la UGBM-INEN durante el periodo 2010-2012. La población del estudio estuvo conformada por pacientes con diagnóstico molecular de LPA PML/RARa. Se incluyeron los pacientes con estudio molecular de la fusión génica PML/RARa por RT-PCR por solicitud del INEN u otras instituciones, se excluyeron pacientes con resultado molecular negativo y pacientes que acudian por monitorización molecular de la enfermedad con resultados previos. El presente estudio no cuenta con una muestra ya que se trabajó con la totalidad de pacientes que cumplieron los criterios mencionados durante el periodo 2010-2012: 50 casos registrados por primera vez en la UGBM-INEN con resultado positivo para la fusión PML/RARa y subtipo molecular por RT-PCR.

De los 50 casos incluidos, solo 28 contaban con historia clínica en el INEN, de donde se obtuvo el informe de mielograma con las características morfológicas de los promielocitos leucémicos según la clasificación Franco-Américo-Británica (FAB) (6) (hipergranulares M3 o hipogranulares M3V) y los valores de leucocitos y plaquetas en sangre periférica al diagnóstico para clasificarlos según el grupo de riesgo de recaída (bajo: leucocitos menor o igual a $10 \times 10^{9} / \mathrm{L}$ y plaquetas mayor a $40 \times 10^{9} / \mathrm{L}$; intermedio: leucocitos menor o igual a $10 \times 10^{9} / \mathrm{L}$ y plaquetas menor o igual a $40 \times 10^{9} / \mathrm{L}$; alto: leucocitos mayor a $10 \times 10^{9} / \mathrm{L}$ ) ${ }^{(7)}$. Los 22 casos restantes fueron referidos de otras instituciones y solo poseían los datos de edad y sexo, lo cual constituye una limitación para nuestro trabajo.

Reacción en cadena de la polimerasa con transcriptasa reversa (RT-PCR). Se determinó la presencia de la fusión génica PML/RARa y subtipos moleculares bcr1, bcr2 y bcr3 utilizando el protocolo estandarizado de RT-PCR y cebadores descritos por Van Dongen (18): reacción de retrotranscripción (RT) $\left(16{ }^{\circ} \mathrm{C}\right.$ por $10 \mathrm{~min} ; 42{ }^{\circ} \mathrm{C}$ por $45 \mathrm{~min} ; \quad 99{ }^{\circ} \mathrm{C}$ por $3 \mathrm{~min}$, y $4{ }^{\circ} \mathrm{C}$ hasta finalizar el paso de RT); luego la PCR $\left(95^{\circ} \mathrm{C}\right.$ por $30 \mathrm{~s}$ ( 1 ciclo); $94{ }^{\circ} \mathrm{C}$ por $30 \mathrm{~s} ; 65^{\circ} \mathrm{C}$ por $60 \mathrm{~s}$, y $72{ }^{\circ} \mathrm{C}$ por $60 \mathrm{~s}$ (35 ciclos), sin requerir extensión final, deteniendo el proceso de PCR a $16^{\circ} \mathrm{C}$ ); y finalmente la PCR anidada en las mismas condiciones que la anterior utilizando $1 \mathrm{uL}$ del producto obtenido previamente. Los cebadores fueron: 
PML-A1: CAGTGTACGCCTTCTCCATCA, PML-A2: CTGCTGGAGGCTGTGGAC, RARA-B: GCTTGTAGATGCGGGGTAGA, PML-C1: TCAAGATGGAGTCTGAGGAGG, PML-C2: AGCGCGACTACGAGGAGAT, RARA-D: CTGCTGCTCTGGGTCTCAAT, RARA-E3': GCCCACTTCAAAGCACTTCT.

Se realizó un análisis descriptivo de la información a través de frecuencias y medidas resumen, según corresponda. El estudio se basa en la descripción de los datos recogidos por la UGBM-INEN de forma rutinaria, en estricto cumplimiento del "Manual de procedimiento del Comité Institucional de Ética en Investigación del INEN".

\section{HALLAZGOS}

De los 50 casos incluidos, 27 fueron varones, el grupo de edad más frecuente fue el de 16 a 40 años (27 personas, 11 de ellos varones). La frecuencia de los subtipos moleculares fue: bcr1 en 31 casos $(62 \%)$, bcr2 en siete casos (14\%) y bcr3 en doce casos (24\%); en el total de casos los tres subtipos predominaron entre los 16-40 años en ambos sexos.

En el análisis de los 28 pacientes con historia clínica en el INEN, se encontró que 14 pacientes tenían un riesgo de recaída intermedio y morfología hipergranular subtipo bcr1. En cambio, de los seis pacientes con riesgo de recaída alto, tres con morfología hipogranular fueron bcr3, y tres con morfología hipergranular fueron bcr1.

Uno de los 28 pacientes poseía sospecha clínica de LPA y criterios hematológicos de riesgo alto de recaída; sin embargo, fue catalogado como una LMA no LPA por sus características citomorfológicas, pero tras el estudio molecular se determinó la presencia de la fusión génica PML/RARa con subtipo molecular bcr3.

\section{DISCUSIÓN}

El presente trabajo muestra una mayor frecuencia de la fusión génica PML/RARa en el grupo etario de 16 a 40 años en ambos sexos, similar a las publicaciones de Douer et al. ${ }^{(2,3)}$ sobre la epidemiología de la LPA en latinos; sin embargo, el predominio de LPA en varones encontrado (54\%) difiere con lo hallado por Douer ${ }^{(3)}$ que reporta igual incidencia en varones y mujeres.

El subtipo molecular más frecuente fue bcr1 (62\%) al igual que en otros estudios en Latinoamérica como Argentina y Uruguay (62\%) ${ }^{(11)}$, México (63\%) e incluso en población no latinoamericana, como la asiática $(67 \%)^{(12)}$. Llama la atención que lo encontrado haya sido superior a lo usualmente reportado en latinos (50$55 \%)^{(4,7,10)}$ que era similar en la población caucásica y estadounidense $(50 \%){ }^{(19)}$. En contraste, se halló que en poblaciones como la India, el subtipo más frecuente es bcr3 ${ }^{(20)}$. Estos datos permiten suponer que existen influencias genéticas o medioambientales, como las migraciones de los antepasados amerindios, que determinarían el punto de corte en el gen PML y los subtipos moleculares de LPA y, por eso, se observan diferencias epidemiológicas de la enfermedad a nivel molecular.

De acuerdo a los 28 casos que contaban con los datos de leucocitos y plaquetas al diagnóstico, así como el informe de mielograma, se encuentra que el subtipo bcr1 estuvo presente en la mayoría de los casos con riesgo de recaída intermedio y morfología hipergranular, y al subtipo bcr3 en la totalidad de casos con riesgo de recaída alto y morfología hipogranular que concuerda con lo mencionado en otras publicaciones ${ }^{(14,16)}$. Sin embargo, estos datos no siempre coinciden con los hallazgos moleculares como en los tres casos que presentaron morfología hipergranular y subtipo molecular bcr3, donde podríamos suponer un buen pronóstico por su característica citomorfológica; sin embargo, la presencia del subtipo bcr3 confiere un mal pronóstico a la LPA ${ }^{(15)}$. El caso fue catalogado por citomorfología como LMA no LPA pero, tras la sospecha clínica, se realizó el estudio de RT-PCR encontrando la fusión génica PML/RARa subtipo bcr3, lo cual muestra la importancia de realizar el estudio molecular para establecer el diagnóstico definitivo de LPA y el subtipo molecular.

En conclusión, el trabajo muestra que el subtipo bcr1 es el más frecuente en la población estudiada que fue referida al INEN para el estudio molecular de LPA PML/ RAR $\alpha$ siendo similar a lo encontrado en otros países de Latinoamérica y, además, existen diferencias entre los subtipos bcr1 y bcr3 de acuerdo a las características citomorfológicas y grupos de riesgo de recaída.

Se espera que la información presentada sirva para dar a conocer que el estudio de la LPA PML/RARa debe realizarse mediante un algoritmo diagnóstico basado en la clínica, en los valores hematológicos, la citomorfología y que debe ser confirmado mediante el estudio molecular por RT-PCR, método que nos permite conocer el subtipo molecular de la fusión génica PML/RARa. Este trabajo podría ser útil para estudios posteriores sobre la relación entre los subtipos moleculares y la remisión, recaída, sobrevida de la LPA y el estudio de mutaciones FLT3-ITD. 
Agradecimientos: al Sr. Raúl Mantilla Quispe, del Departamento de Investigación y Docencia del INEN, por la ayuda brindada en el procesamiento de datos.

Contribuciones de autoría: MCCM ha participado en la concepción del artículo. Todos los autores participaron en la recolección de datos, análisis e interpretación de datos, redacción, revisión del artículo y aprobación de la versión final.

Financiamiento: autofinanciado.

Conflictos de interés: los autores niegan tener conflictos de interés.

\section{REFERENCIAS BIBLIOGRÁFICAS}

1. Swerdlow SH, Campo E, Harris NL, Jaffe ES, Pileri SA, Stein H, et al. WHO Classification of Tumours of Haematopoietic and Lymphoid Tissues. Lyon: IARC; 2008.

2. Douer D, Preston-Martin S, Chang E, Nichols PW, Watkins KJ, Levine AM. High frequency of acute promyelocytic leukemia among Latinos with acute myeloid leukemia. Blood. 1996;87(1):308-13.

3. Douer D. The epidemiology of acute promyelocytic leukaemia. Best Pract Res Clin Haematol. 2003;16(3):357-67.

4. Rego EM, Jácomo RH. Epidemiology and treatment of acute promyelocytic leukemia in Latin America. Mediterr J Hematol Infect Dis. 2011;3(1):e2011049.

5. Perú, Instituto Nacional de Enfermedades Neoplásicas (INEN). Perfil epidemiológico [Internet]. Lima: INEN; c2013 [citado el 5 de enero de 2013]. Disponible en: http://www.inen.sld.pe/ portal/estadisticas/datos-epidemiologicos.html

6. Bennett JM, Catovsky D, Daniel MT, Flandrin G, Galton DA, Gralnick HR, et al. Proposed revised criteria for the classification of acute myeloid leukemia: A report of French-American-British Cooperative Group. Ann Intern Med. 1985;103(4):620-5.

7. Sanz MA, Lo Coco F, Martín G, Avvisati G, Rayón C, Barbui T, et al. Definition of relapse risk and role of nonanthracycline drugs for consolidation in patients with acute promyelocytic leukemia: a joint study of the PETHEMA and GIMEMA cooperative groups. Blood. 2000;96(4):1247-53.

8. Rowley JD, Golomb HM, Dougherty C. 15/17 translocation, a consistent chromosomal change in acute promyelocytic leukaemia. Lancet. 1977;1(8010):549-50.

9. Borrow J, Goddard AD, Gibbons B, Katz F, Swirsky D, Fioretos T, et al. Diag- nosis of acute promyelocytic leukaemia by RT-PCR: detection of PML-RARAPML fusion transcripts. Br J Haematol. 1992;82(3):529-40.

10. Douer D, Santillana S, Ramezani L, Samanez C, Slovak ML, Lee MS, et al. Acute promyelocytic leukaemia in patients originating in Latin America is associated with an increased frequency of the bcrl subtype of the PML/ RARalpha fusion gene. Br J Haematol. 2003;122(4):563-70.

11. Uriarte MA, Zubillaga MNZ, Chacon AC, Bononi RB, Lombardi VL, Giordano HG, et al. Genetic Characterization and Follow-up of MRD in Acute Promyelocytic Leukemia Patients from Argentina and Uruguay. Haematologica Reports. 2005;1:47.

12. Ruiz-Argüelles GJ, Garcés-Eisele J Reyes-Núñez V, Gómez-Rangel JD, Ruiz-Delgado GJ. More on geographic hematology: the breakpoint cluster regions of the PML/RAR $\alpha$ fusion gene in Mexican Mestizo patients with promyelocytic leukemia are different from those in Caucasians. Leuk Lymphoma. 2004;45(7):1365-8.

13. Arrigoni P, Beretta C, Silvestri D, Rossi V, Rizzari C, Valsecchi MG, et al. FLT3 internal tandem duplication in childhood acute myeloid leukaemia: association with hyperleucocytosis in acute promyelocytic leukaemia. Br J Haematol. 2003;120(1):89-92.

14. Foley R, Soamboonsrup P, Carter RF, Benger A, Meyer R, Walker I, et al. CD34-positive acute promyelocytic leukemia is associated with leukocytosis, microgranular/hypogranular morphology, expression of CD2 and bcr3 isoform. Am J Hematol. 2001;67(1):34-41.

15. Yoo SJ, Park CJ, Jang S, Seo E-J, Lee K-H, Chi HS. Inferior prognostic outcome in acute promyelocytic leukemia with alterations of FLT3 gene. Leuk Lymphoma. 2006;47(9):1788-93.
16. Jácomo RH, Figueiredo-Pontes LL de, Rego EM. Do paradigma molecular ao impacto no prognóstico: uma visão da leucemia promielocítica aguda. Rev Assoc Med Bras. 2008;54(1):82-9.

17. Santamaría C, Chillón MC, Fernández C, Martín-Jiménez P, Balanzategui A, García Sanz R, et al. Using quantification of the PML-RARalpha transcript to stratify the risk of relapse in patients with acute promyelocytic leukemia. Haematologica. 2007;92(3):315-22

18. van Dongen JJ, Macintyre EA, Gabert JA, Delabesse E, Rossi V, Saglio G, et al. Standardized RT-PCR analysis of fusion gene transcripts from chromosome aberrations in acute leukemia for detection of minimal residual disease Report of the BIOMED-I Concerted Action: Investigation of minimal residual disease in acute leukemia. Leukemia. 1999;13(12):1901-28.

19. Roosevelt AC, Douglas J, Brown L. The migrations and adaptations of the first Americans: Clovis and pre-Clovis viewed from South America. In: The First Americans: The Pleistocene Colonization of the New World. San Francisco, CA: California Academy of Sciences; 2002. p. 159-235.

20. Sazawal S, Hasan SK, Dutta P, Kumar B Kumar R, Kumar L, et al. Over-representation of bcr3 subtype of PML/RAR $\alpha$ fusion gene in APL in Indian patients. Ann Hematol. 2005;84(12):781-4.

Correspondencia: Maria del Carmen Castro Mujica

Dirección: Av. Angamos Este 2520. Lima 34, Perú.

Teléfono: (511) 201-6500; Anexo 3007.

Correo electrónico:maria.castro.m@upch.pe 